\title{
087
}

\section{VALUTAZIONE PRELIMINARE DEL SISTEMA CHORUS PER L'ANALISI DELL'AVIDITÀ DELLE IgG ANTI TOXOPLASMA GONDII}

\author{
Mazzarelli G., Parri F. \\ Laboratorio di Sieroimmunologia, A.O. U.C. Careggi, \\ Viale Pieraccini I, Firenze.
}

Introduzione. La misura dell'avidità delle IgG è un test particolarmente utile nella sierologia dell'infezione da Toxoplasma gondii, soprattutto in virtù del fatto che la sola ricerca delle IgM, IgG e IgA anti Toxoplasma spesso non è sufficiente per datare l'infezione.

Nel presente lavoro abbiamo valutato il kit "Chorus Toxoplasma IgG avidità" applicato al sistema Chorus (Diesse Diagnostica Senese Spa, Monteriggioni) nei confronti del kit "Enzywell Toxoplasma IgG avidità" (Diesse Diagnostica Senese Spa, Monteriggioni) in uso presso il nostro laboratorio.

Materiali e metodi. Sono stati analizzati 85 campioni di routine al fine di valutare la concordanza tra i due metodi sottoposti a confronto. I casi discordanti sono stati ulteriormente investigati con i metodi di immunofluorescenza totale, immunofluorescenza IgM (test di Remington) ed il test per la ricerca delle IgA specifiche, nonché, ove possibile, confrontando i risultati ottenuti in prelievi dallo stesso paziente, precedentemente o successivamente sottoposti ad analisi sierologiche per toxoplasmosi.

Risultati. I due test a confronto sono risultati concordare in 78 casi su 85 (91,8\%): in particolare 57 campioni hanno mostrato alta avidità sia con il sistema Chorus sia con il kit Enzywell, 18 un valore di bassa avidità con entrambi i metodi e solo 3 hanno prodotto un risultato dubbio.

Per quel che riguarda i 7 campioni discordanti, 3 hanno evidenziato un valore di alta avidità con Chorus ma media avidità con Enzywell, 3 alta avidità con Chorus ma bassa avidità con Enzywell e solo uno bassa avidità con Chorus ma alta avidità con Enzywell.

Conclusioni. Le ulteriori analisi cui sono stati sottoposti i 7 campioni discordanti hanno evidenziato una congruenza con il valore di avidità ottenuto con il sistema Chorus del 71\% (5 casi su 7), che si è perciò dimostrato più attendibile rispetto 
alla metodica in micropiastra. È stato inoltre riscontrato che i valori percentuali che si riscontrano con il test Enzywell Toxoplasma IgG avidità (per i casi di infezione pregressa) risultano, a differenza dei valori riscontrati con il sistema Chorus, poco discriminanti in quanto si avvicinano troppo alla soglia dei dubbi. 